\title{
INFLUENCIA DE LA SOMBRA Y DE LAS MICORRIZAS SOBRE EL CRECIMIENTO DE PLANTAS DE LULO (Solanum quitoense Lam.)
}

\section{INFLUENCE OF SHADING AND MYCORRHIZAE ON GROWTH OF LULO PLANTS (Solanum quitoense Lam.)}

\author{
Fánor Casierra-Posada ${ }^{1}$; Jaime Peña-Olmos ${ }^{2}$; Juan Peñaloza ${ }^{3}$; Gabriel Roveda ${ }^{4}$
}

\begin{abstract}
${ }^{1}$ I.A. Ph.D., profesor asociado Facultad de Ciencias Agropecuarias de la Universidad Pedagógica y Tecnológica de Colombia. Tunja / Colombia. Grupo de Investigación Ecofisiología Vegetal. E-mail: fanor.casierra@uptc.edu.co [Autor para correspondencia]; ${ }^{2}$ I.A., Estudiante de Maestría en Ciencias Biológicas UPTC. E-mail: jaime.pena@uptc.edu.co; ${ }^{3}$ I.A. UPTC. E-mail: jdiegops83@hotmail.com; ${ }^{4}$ I.A., M.Sc. Grupo de Recursos Biofísicos. Corpoica, C.I. Tibaitatá. E-mail: groveda@gmail.co
\end{abstract}

Rev. U.D.C.A Act. \& Div. Cient. 16(1): 61 - 70, 2013

\section{RESUMEN}

El lulo es originario de los bosques andinos, donde crece bajo sombra parcial, asociado a micorrizas nativas. Se han establecido cultivos comerciales en ambientes de alta iluminación, lo que genera estrés. Para evaluar el crecimiento de plantas de lulo inoculadas con micorrizas y bajo condiciones de sombreado, se estableció un estudio en invernadero en Tunja, Colombia. Las plantas, se inocularon con Mycobiol ${ }^{\circledR}$ (combinación de Glomus sp., Entrophospora colombiana y Acaulospora mellea) y con Scutellospora heterogama, Acaulospora mellea y Glomus spp., individualmente. Sobre la mitad de las plantas micorrizadas, se extendió una malla polisombra. A las plantas control no se les aplicaron micorrizas ni sombra. Tanto Glomus sp. como S. heterogama indujeron plantas $55 \%$ más altas que aquellas que no habían sido micorrizadas. Las plantas, en pleno sol tratadas con $S$. heterogama, Mycobiol, Glomus sp. y A. mellea, presentaron, respectivamente, $290 \%, 186 \%, 142 \%$ y $124,2 \%$ más área foliar que las plantas control. Se produjo una reducción de $37 \%$ en el peso específico de las hojas en plantas expuestas a la sombra, en relación con las plantas micorrizadas a plena exposición. Bajo sombra, las plantas disminuyeron $27 \%$ la acumulación de materia seca en relación con las plantas micorrizadas a libre exposición. Las plantas a pleno sol inoculadas con S. heterogama y con Glomus sp. produjeron $153 \%$ y $132 \%$ más materia seca que las plantas control. La inoculación con micorrizas compensó el efecto negativo de la sombra sobre el crecimiento de las plantas.

Palabras clave: Sombreado, área foliar, peso específico de las hojas, materia seca.

\section{SUMMARY}

The lulo plant (Solanum quitoense) native to Andean forests grows under partial shade in association with native mycorrhizae. In commercial cultivation lulo is often planted in full sun, which leads to plant stress. In order to evaluate the growth of lulo plants inoculated with mycorrhizae, under partial shade conditions, a greenhouse study was CARRIED OUT in Tunja, Colombia. The plants were inoculated with Mycobiol ${ }^{\circledR}$ (a combination of Glomus spp., Entrophospora colombiana, and Acaulospora mellea), Scutellospora heterogama alone, Acaulospora mellea alone, and Glomus spp. alone. Half of these inoculated plants were grown under a shade cloth, while the control plants were grown in full sun without mycorrhizae. Glomus spp. and S. heterogama induced full-sun plants $55 \%$ taller than in treatments without mycorrhizae. Full-sun plants treated with $S$. heterogama. Mycobiol, Glomus spp., and A. mellea respectively presented $290 \%, 186 \%, 142 \%$, and $124 \%$ more leaf area than plants without mycorrhizae. There was a 37\% reduction in specific leaf weight in shaded plants with respect to mycorrhizal plants in full sun. Shaded mycorrhizal plants also had $27 \%$ lower dry matter accumulation compared to plants in full sun. Fullsun plants inoculated with S. heterogama and with Glomus spp. produced $153 \%$ and $132 \%$ more dry matter than control plants. Inoculation with mycorrhizae compensated the negative effect of shade on plant growth.

Key words: Shading, leaf area, specific leaf weight, dry matter. 


\section{INTRODUCCIÓN}

El lulo (Solanum quitoense Lam.) crece en forma espontánea en el área andina, en especial, bajo condiciones de sotobosque, en sitios frescos, sombreados, cercanos a corrientes de agua, con temperaturas entre 17 y $20^{\circ} \mathrm{C}$ (Medina et al. 2008); además, es una especie que, frecuentemente, interactúa con hongos formadores de micorrizas arbusculares. Aunque se puede cultivar bajo sombrío, su cultivo a plena exposición reduce, de manera considerable, el período productivo (Lobo, 2000), como consecuencia de la actividad fotosintética y de un metabolismo acelerado, lo que conlleva al debilitamiento ante ataques subsiguientes de plagas y de enfermedades (Lobo, 1991). Por tanto, las condiciones de sotobosque conllevan a una reducción sustancial de la cantidad de radiación incidente sobre las plantas de lulo, las cuales, deben aprovechar la radiación difusa, en gran medida.

La micorriza es una asociación mutualista entre un hongo y una planta superior (Menge, 1983). Pueden existir diferentes tipos de micorrizas, cuyas diferencias radican en su morfología y su fisiología (Turk et al. 2006). En la asociación micorriza/hospedero, el hongo ofrece aminoácidos, nutrientes y agua al hospedero, a cambio de fotosintatos (Smith \& Read, 1997). Debido a que la micorriza se comporta como un vertedero de carbohidratos, se altera tanto la fotosíntesis como la distribución de carbohidratos en la planta hospedera (Nehls \& Hampp, 2000; Wright et al. 2000), por tanto, los cambios en la radiación incidente (McGee \& Furby, 1992) y la calidad del espectro (Rosa et al. 1999) pueden afectar la asociación hospedero micorriza.

Existen reportes en que el sombreado reduce la infección en endomicorrizas (Boullard, 1960); sin embargo, Baylis (1967) encontró que el sólo sombreado no reduce la infección de Coprosma robusta en un suelo de bosque. Las plántulas micorrizadas de especies tolerantes a la sombra presentaron menor infección cuando crecían en ambientes fuertemente sombreados, en comparación que cuando se encontraban a plena exposición solar; mientras que las especies de plantas con alta demanda de luz presentaban altos niveles de infección por parte del hongo y acumularon fósforo, incluso, bajo condiciones de fuerte sombreado (Johnson, 1976).

Rosa et al. (1998) estudiaron el crecimiento de plántulas fertilizadas de Pinus sylvestris bajo diferentes fuentes de luz de amplio espectro y encontraron que la radiación, con baja relación rojo/rojo lejano, reducía el número de raíces cortas y el desarrollo de las micorrizas, lo cual, atribuyen al resultado de la reducción en el envío de fotosintatos a las raíces; no obstante, los autores no detectaron efecto complementario de la radiación rojo lejano sobre la formación de las micorrizas, ni frecuencias morfotípicas en plántulas sin fertilización. También, se ha estudiado el efecto de la radiación de amplio espectro sobre la interacción entre ectomicorrizas y $P$. sylvestris y se encontró que el hongo creció sobre las raíces laterales, pero no desarrolló la red de Hartig en los tratamientos con luz; además, el efecto de la radiación de amplio espectro sobre la interacción hongo/raíz fue altamente dependiente del estado de desarrollo de la raíz (Niemi et al. 2005).

En diversos estudios se ha constatado el beneficio ofrecido por el sombrío sobre algunas plantas cultivadas comercialmente, como Coffea arabica (Cardona \& Sadeghian, 2005). Bajo estas condiciones, los cafetos incrementan su tasa fotosintética (Jaramillo \& Gómez, 1989); sin embargo, es poco lo que se conoce con respecto al efecto del sombrío sobre las poblaciones de hongos micorrízicos sobre los vegetales. Se ha encontrado que el sistema de producción a libre y a mediana exposición indujo un incremento del micelio, determinado en épocas de máximo verano, dado que se incrementa el brillo solar, la temperatura y disminuyen las precipitaciones; en consecuencia, la humedad del suelo se reduce, así como la disponibilidad de nutrientes (Swisher, 1999; Paz \& Sánchez, 2007).

Las especies frutícolas perennes o semiperennes requieren una etapa de vivero antes del establecimiento del huerto comercial (Alarcón \& Ferrera-Cerrato, 1999). Es, precisamente, en la etapa de vivero o en las etapas tempranas de la planta, en donde la aplicación e inoculación de micorrizas representa un gran potencial para mejorar el crecimiento y el desarrollo (Roveda et al. 2007), ya que la micorriza actúa como acelerador del crecimiento, por lo que se pueden obtener plantas con mayor vigor y sanidad. Otro beneficio correspondería a la reducción de costos de producción en función de la reducción de la aplicación de fuentes fertilizantes (Alarcón \& Ferrera-Cerrato, 1999), como consecuencia de la alta capacidad de la planta micorrizada para tomar nutrientes, en especial, el fósforo. Se reporta que una de las principales estrategias de las plantas para adaptarse a condiciones adversas de suelo es mediante el incremento de la superficie radical, a través de las micorrizas (Marschner, 1991).

El objetivo de presente estudio consistió en la evaluación del crecimiento en plantas de lulo (S. quitoense), que habían sido inoculadas con diferentes cepas de micorrizas y expuestas a condiciones de sombrío y a plena exposición, bajo condiciones de invernadero.

\section{MATERIALES Y MÉTODOS}

El ensayo, se realizó en el invernadero de la Facultad de Ciencias Agropecuarias de la Universidad Pedagógica y Tecnológica de Colombia (UPTC). Como material vegetal, se utilizaron plántulas obtenidas a partir de semilla de lulo ( $S$. quitoense var. bot. septentrionale), provenientes del laboratorio de cultivo de tejidos Bioplasma de la UPTC, las cuales, 
se transplantaron a bolsas, con capacidad para cuatro kg, que contenían tierra negra, proveniente de un Andosol de la región.

El inóculo utilizado en el experimento fue suministrado por el Programa Nacional de Recursos Biofísicos de CORPOICA, C.I. Tibaitatá. El producto consistía en una mezcla de suelo, de hifas, de esporas de la micorriza y de raíces colonizadas. Los tipos de micorriza utilizados correspondieron a Acaulospora mellea, Scutellospora hetereogama, Glomus sp., el producto comercial Mycobiol ${ }^{\circledR}$ (Corpoica), el cual, contiene tres tipos diferentes de micorrizas de los géneros Glomus sp., Entrophospora colombiana, Acaulospora mellea. Las plantas control no se inocularon con ningún tipo de micorriza. La inoculación de las plantas con la micorriza, se realizó al momento del transplante del semillero a las bolsas de cuatro $\mathrm{kg}$, colocando una cantidad del producto que contenía las micorrizas, de manera que cerca de las raíces quedara una cantidad aproximada a 200 esporas del hongo.

Para efectos del sombreado, se cubrieron un grupo de plantas con malla polisombra negra, que indujo una reducción de $24,9 \%$ con respecto a las plantas a libre exposición, dentro del invernadero. La iluminación medida correspondió a $782,2 \mu \mathrm{mol} . \mathrm{m}^{-2} \cdot \mathrm{s}^{-1} \mathrm{y}$, bajo la malla polisombra, se registró una iluminación de 587,0 $\mu \mathrm{mol} . \mathrm{m}^{-2} . \mathrm{s}^{-1}$. La radiación registró en lx con un luxómetro Testoterm 0500® (Alemania) y fue convertida a $\mu \mathrm{mol} \cdot \mathrm{m}^{-2} \cdot \mathrm{s}^{-1}$.

Como variables respuesta, se determinaron el peso seco, por secado en estufa a $105^{\circ} \mathrm{C}$ hasta peso constante; la distribución de la materia seca en los diferente órganos, como la cantidad de peso seco a cada órgano con respecto al peso seco total de la planta; el peso especifico de las hojas, como el cociente entre el área foliar y el peso seco de las hojas; la tasa de crecimiento absoluto, relativo y de asimilación neta, de acuerdo con las formulas de Hunt (1990); la altura de la planta medida con un flexómetro; el área foliar determinada con un medidor integrado Li-cor 3000A® (USA); la relación raíz/vástago, como el cociente entre el peso seco de la raíz y el peso de la parte aérea. Al final del ensayo, en el suelo donde crecieron las plantas, se determinó el número de esporas por gramo de suelo, mediante el procedimiento descrito por Gerdemann \& Nicolson (1963).

El ensayo consistió en un experimento bifactorial, donde el primer factor era el sombreado y, el segundo, estaba constituido por el tipo de micorriza (sin micorriza, Mycobiol, Scutellospora heterogama, Acaulospora mellea, Glomus sp.), organizados en un diseño, el bloque al azar, con diez replicaciones. La unidad experimental estuvo constituida por siete plantas. Para el análisis estadístico, se hizo una prueba de variancia, mediante una tabla ANAVA $(P<0,05)$ y la prueba de separación de promedios de Tukey. Estas pruebas, se rea- lizaron con la ayuda de la aplicación SPSS $®$ versión 11.5.1 (Statistical Product and Service Solutions, Chicago, Illinois, USA - Nov. 2002).

\section{RESULTADOS Y DISCUSIÓN}

Se encontró diferencia altamente significativa $(P<0,01)$ para los factores sombra y micorrizas, así como para todas sus interacciones (sombra x micorrizas), en todas las variables evaluadas, con excepción del número de esporas por gramo de suelo seco y de la tasa de crecimiento relativo, en la cual, hubo diferencia para el factor sombra y para la interacción sombra x micorrizas, pero no para el factor micorrizas. En lo relacionado con la altura de la planta, la sombra motivó la elongación de las plantas en un $11,6 \%$ en relación con las plantas plena exposición. Independientemente del sombreado, las micorrizas que en mayor grado motivaron la elongación de las plantas fueron Glomus sp. y S. heterogama, que indujeron plantas $54,7 \%$ más altas que aquellas que no habían sido micorrizadas (control) (Tabla 1). De igual manera, Siqueira et al. (1998) encontraron un incremento en la altura y en el diámetro del tallo en plantas de Coffea sp., como consecuencia de la inoculación de las plantas con esporas de Glomus clarum, Gigaspora margarita y Glomus etunicatum. Asimismo, Pereira et al. (2001) reportan que las plantas de Eucalyptus camaldulensis presentan mayor altura de plántulas cuando se inoculan con Glomus intraradices, pero no así con G. mosseae, como consecuencia de un mayor grado de micorrización alcanzada por las plantas inoculadas con $G$. intraradices $(86,7 \%)$, con su consiguiente aumento en la absorción de agua y de nutrientes para la planta.

Las plantas expuestas al sombreado presentaron $56,8 \%$ más área foliar que las que se encontraban a libre exposición, con diferencias estadísticamente significativas, lo cual, está relacionado con un mecanismo de adaptación de las plantas a las condiciones del sombreado. Las plantas sufren variaciones en el tamaño del follaje, en la densidad y en la posición de los estomas, debido a la necesidad que tienen de adaptarse a las condiciones de luz (Sprugel et al. 1996). Mientras a libre exposición, las ramas y las hojas sirven de protección de los tallos contra la radiación directa, bajo sombra, el factor limitante es la falta de luz, a lo cual, las plantas responden produciendo hojas de mayor área, con un mesófilo poco desarrollado. Una mayor superficie foliar ayuda a las plantas a capturar un mayor número de fotones (Schulze et al. 2002); sin embargo, esta efectividad en la captación de luz no implica que las plantas bajo sombra sean más eficientes que las plantas a plena exposición, en cuanto a la utilización de los fotoasimilados. En relación con las micorrizas, sin tener en cuenta la sombra, todas las plantas tratadas con los hongos micorrizógenos presentaron mayor área foliar que las plantas control. Respectivamente, las plantas tratadas con S. heterogama. Mycobiol, Glomus sp., y A. mellea, presen- 
Tabla 1. Área foliar, peso específico de las hojas y altura de la planta en plantas de lulo (Solanum quitoense Lam.), inoculadas con diferentes especies de micorrizas y expuestas a dos niveles de iluminación.

\begin{tabular}{|c|c|c|c|c|c|c|c|c|c|c|}
\hline \multirow{3}{*}{ Iluminación } & \multirow{3}{*}{ Micorriza } & \multicolumn{9}{|c|}{ Variable } \\
\hline & & \multicolumn{3}{|c|}{ Área foliar $\left(\mathrm{cm}^{2}\right)$} & \multicolumn{3}{|c|}{$\begin{array}{l}\text { Peso especifico de las } \\
\text { hojas }\left(\mathrm{mg} \mathrm{cm}^{-2}\right)\end{array}$} & \multicolumn{3}{|c|}{$\begin{array}{l}\text { Altura de la planta } \\
\text { (cm) }\end{array}$} \\
\hline & & Media & Desv. & Tukey & Media & Desv. & Tukey & Media & Desv. & Tukey \\
\hline \multirow{5}{*}{$\begin{array}{l}\text { Plena ex- } \\
\text { posición }\end{array}$} & Control & 226,05 & 28,62 & $\mathrm{a}$ & 5,17 & 0,66 & def & 15,77 & 1,33 & a \\
\hline & Mycobiol & 573,03 & 162,58 & bc & 5,30 & 1,33 & ef & 18,18 & 1,68 & $\mathrm{~b}$ \\
\hline & S. heterogama & 507,34 & 100,02 & $\mathrm{~b}$ & 5,94 & 0,82 & $\mathrm{f}$ & 28,11 & 1,82 & de \\
\hline & A. mellea & 614,39 & 120,95 & bc & 4,91 & 1,09 & de & 26,10 & 1,86 & $\mathrm{~d}$ \\
\hline & Glomus sp. & 596,46 & 42,72 & bc & 4,37 & 0,74 & $\mathrm{~cd}$ & 28,21 & 1,36 & de \\
\hline \multirow{6}{*}{ Sombrío } & Control & 294,60 & 54,29 & $a$ & 3,48 & 0,67 & bc & 22 & 2,35 & $\mathrm{c}$ \\
\hline & Mycobiol & 916,15 & 88,40 & d & 2,67 & 0,52 & $a b$ & 21,04 & 1,82 & c \\
\hline & S. heterogama & 1521,67 & 98,61 & $\mathrm{e}$ & 2,06 & 0,16 & $a$ & 30,28 & 2,70 & $\mathrm{e}$ \\
\hline & A. mellea & 552,95 & 172,84 & bc & 4,401 & 1,34 & cde & 26,41 & 2,33 & d \\
\hline & Glomus sp. & 663,80 & 100,50 & c & 3,49 & 0,55 & b & 30,16 & 2,99 & e \\
\hline & C.V. (\%) & & 55,57 & & & 34,62 & & & 21,32 & \\
\hline
\end{tabular}

Promedios con letras iguales no presentan diferencias significativas según la prueba de Tukey $(P<0,01)$

taron 289,8\%; $186 \%$; $142 \%$ y 124,2\% más área foliar que las plantas sin tratamiento adicional con micorrizas (control) (Tabla 1). De igual manera, se encontró un incremento en el área foliar en plantas de Citrus volkameriana inoculadas con Glomus intraradices (Peng et al. 1993). Este incremento significativo del área foliar en plantas micorrizadas es el resultado de una mayor demanda de carbohidratos por parte del hongo micorrizógeno, carbohidratos que la planta sintetizará partiendo de una mayor capacidad en la interceptación de fotones. Por tal razón, la planta, en presencia de micorrizas arbusculares, tiende a desarrollar más su área foliar, con el fin de ser más eficiente en la captación de luz y abastecer las demandas de fotoasimilados por parte de la misma y del micosimbionte. Harris et al. (1985) evaluaron la inoculación individual y doble de Rhizobium y hongos formadores de micorrizas del genero Glomus, en plantas de soya. A las seis semanas de crecimiento, se encontró que los nódulos en plantas micorrizadas consumían un $12 \%$ de los fotosintatos, mientras que la micorrizas consumían un $17 \%$. El contenido de almidón de las hojas en las plantas noduladas y micorrizadas había descendido en $50 \%$, debido al requerimiento de los dos simbiontes. Este alto consumo de la doble simbiosis se vio compensado por un incremento del $47 \%$, en las tasas de fijación del $\mathrm{CO}_{2}$, con respecto a los controles no inoculados y un aumento del $24 \%$, en el área foliar específica. El área foliar y el ritmo de fijación de carbono por unidad de superficie en las plantas de soya micorrizadas y noduladas fue superior con respecto a los controles nodulados y adicionados con fósforo, razón por la cual, la asimilación de carbono en dichas plantas aumentó (Gianinazzi-Pearson \& Azcón-Aguilar, 1991).

Se produjo una reducción del orden de 37,3\% en el peso específico de las hojas en plantas expuestas a la sombra, versus plantas a plena exposición. En cuanto a las micorrizas, el valor presentado el control solamente fue superado por A. mellea, con $6,9 \%$, mientras que $S$. heterogama presentó $6,9 \%$ menos y, a su vez, Glomus sp. y Mycobiol, mostraron 9,3\% menos que las plantas control (Tabla 1). Hepper (1977) reportó un incremento del $16 \%$ en el peso seco de la raíz en plantas micorrizadas. Esto implica una mayor demanda de fotosintatos por parte del hongo, los cuales, son transportados desde la parte aérea, principalmente desde las hojas, hacia la raíz de la planta. Las raíces micorrizadas consumen entre 4 y $20 \%$ más del carbono fijado en la fotosíntesis, en comparación con las raíces no micorrizadas, siendo la planta compensada por incrementos en la tasa fotosintética, debido a elevadas concentraciones de fósforo foliar (Jakobsen, 1995).

Para la variable peso seco total acumulado por planta, aquellas expuestas a la sombra, disminuyeron la acumulación de materia seca, en un $27,3 \%$, en relación con las plantas a libre 
exposición. Generalmente, las plantas expuestas a pleno sol presentan hojas que, por lo regular, son más gruesas y tienen una mayor masa por área que las que se desarrollan bajo condiciones de sombra (Sprugel et al. 1996). En general, las plantas inoculadas con S. heterogama produjeron 152,7\% más materia seca que las plantas control, de igual manera, Glomus sp. produjo $131,8 \%$ más, A. mellea 114,6\% y Mycobiol 106,9\% (Figura 1). Este incremento en la acumulación de biomasa es una consecuencia de un mayor flujo de nutrientes minerales (principalmente fósforo) hacia el hospedero, debido, fundamentalmente, a la presencia de micorrizas arbusculares (Sánchez de Prager, 1999).

En cuanto al peso seco en hojas, en tallos y en raíces, se observó una desproporción en la distribución de las plantas no micorrizadas. El testigo con sombra mostró una dismi-

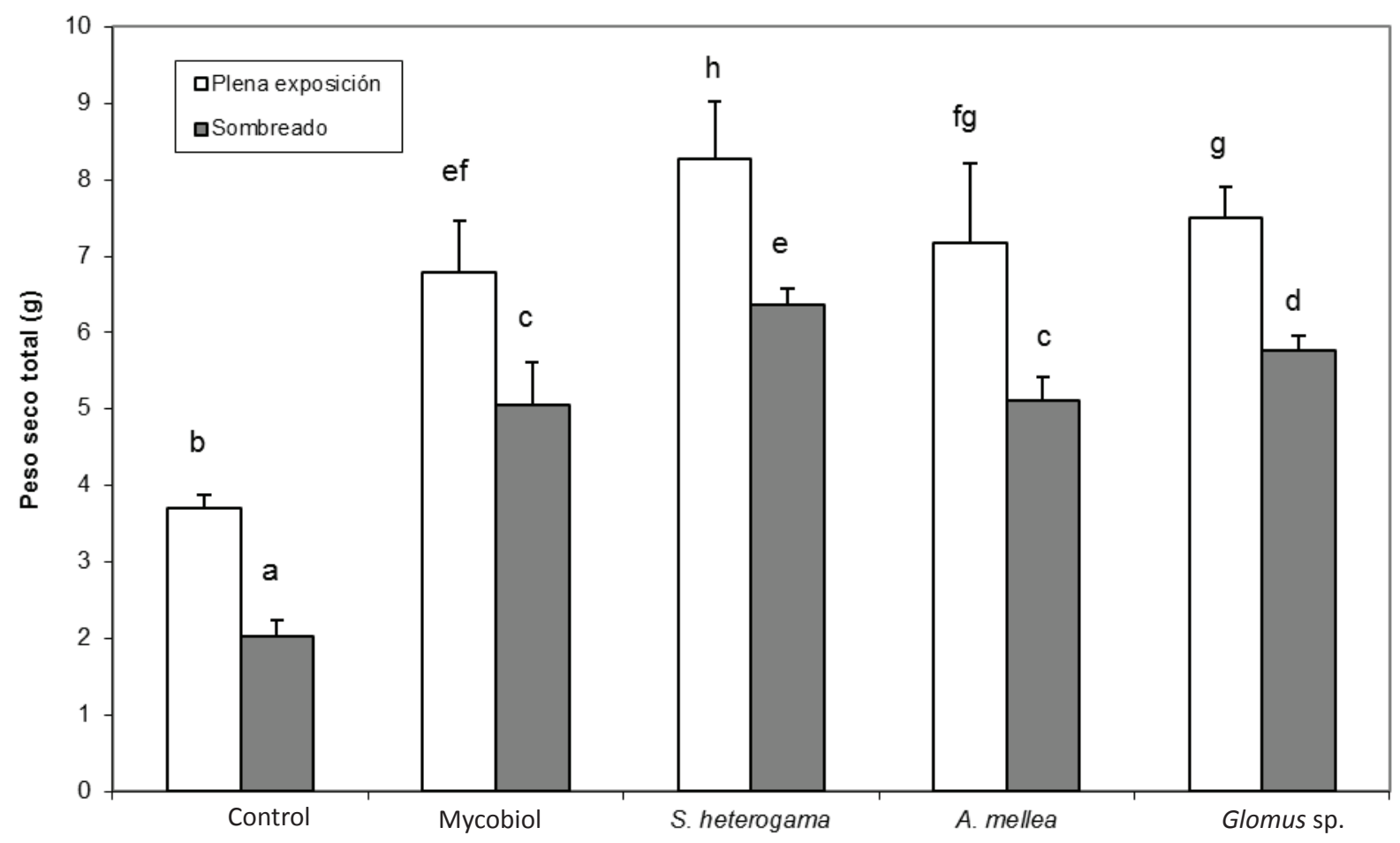

Especie de micorriza

Figura 1. Producción total de peso seco en plantas de lulo (Solanum quitoense Lam.), inoculadas con diferentes especies de micorrizas y expuestas a dos niveles de iluminación.

nución del $19,44 \%$ en el peso seco de la raíz con respecto a S. heterogama, 33,37\% menos que Mycobiol, 35,1\% menos que Glomus sp. y 36,9\% menos que A. mellea, todos bajo sombra. En la parte aérea el testigo con sombra presentó el mayor porcentaje de peso seco, tanto para hojas $(49,64 \%)$ como para tallos (25,83\%), lo cual, muestra una planta poco homogénea, condición que no se dio en los tratamientos micorrizados bajo sombra (Figura 2). Por otra parte, el testigo sin sombra evidenció una mayor acumulación de su peso seco en la raíz, con un $29,5 \%$ más que $S$. heterogama, 30,15\% sobre Glomus sp., 31,2\% sobre Mycobiol y 45,56\% más que $A$. mellea. Contrario a lo que sucedió con su parte aérea, en donde el testigo sin sombra mostró el menor desarrollo, con $31,18 \%$, para hojas y $9,4 \%$, para tallos, otra muestra de un cambio en el patrón de distribución de ma- teria seca, en este caso, a favor del peso seco de la raíz, la que nuevamente se vio más homogénea en los tratamientos inoculados con micorrizas y a libre exposición (Figura 2).

La relación raíz/vástago mostró diferencias a favor de las plantas a libre exposición, con un 36,58\% más que las plantas influenciadas por la sombra, evidenciándose un mayor desarrollo radicular en las primeras. El factor micorrizas presentó diferencias significativas a favor de las plantas no micorrizadas, que presentaron un incremento del $26,78 \%$, con respecto a las plantas inoculadas con Glomus sp., 28,33\% por encima de Mycobiol, 35,49\% más que S. heterogama y $37,72 \%$ más con respecto a A. mellea (Figura 3). Lo anterior concuerda con lo señalado por Sánchez de Prager (1999), quien afirma que el establecimiento y el buen desarrollo de 


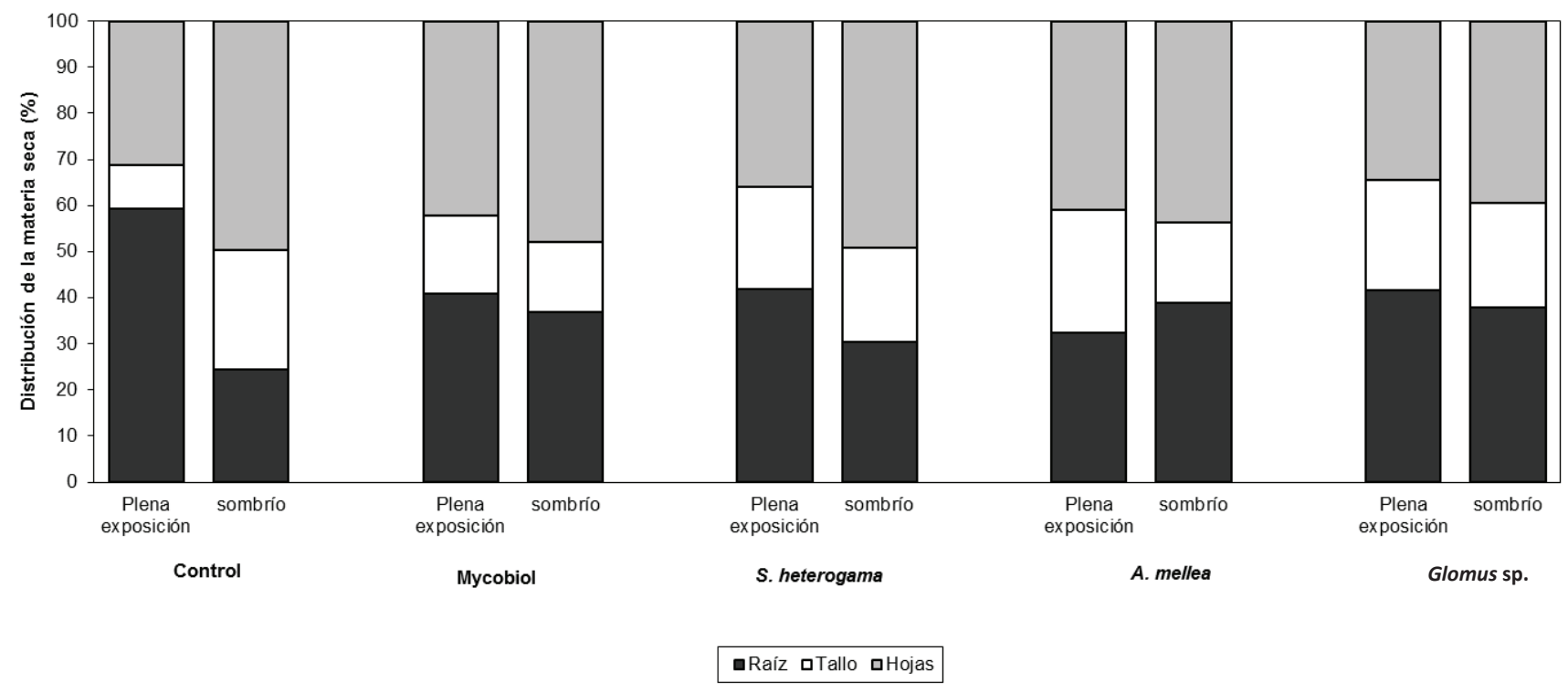

Figura 2. Distribución porcentual de materia seca en raíces, en tallo y en hojas en plantas de lulo (Solanum quitoense Lam.), inoculadas con diferentes especies de micorrizas y expuestas a dos niveles de iluminación.

la simbiosis, además de incrementar la biomasa vegetal tiene una marcada influencia en la distribución de la misma, debido a una mayor translocación de nutrimentos desde la raíz hacia la parte aérea de la planta, la cual, propicia, relativamente, una menor transferencia de fotosintatos a la raíz y una mayor retención de éstos en la parte aérea, donde la planta los utiliza para producir material vegetal. Es por esta razón, que la relación raíz/vástago, generalmente, es más baja en plantas micorrizadas.

La tasa de crecimiento relativo (TCR) se vio influenciada por el factor sombra, pero no por las micorrizas, es así, como la sombra redujo el valor de esta variable, en 29,4\% (Figura 4). De igual manera, Guarnaschelli et al. 2006, investigaron las posibles diferencias en el comportamiento de dos clones de

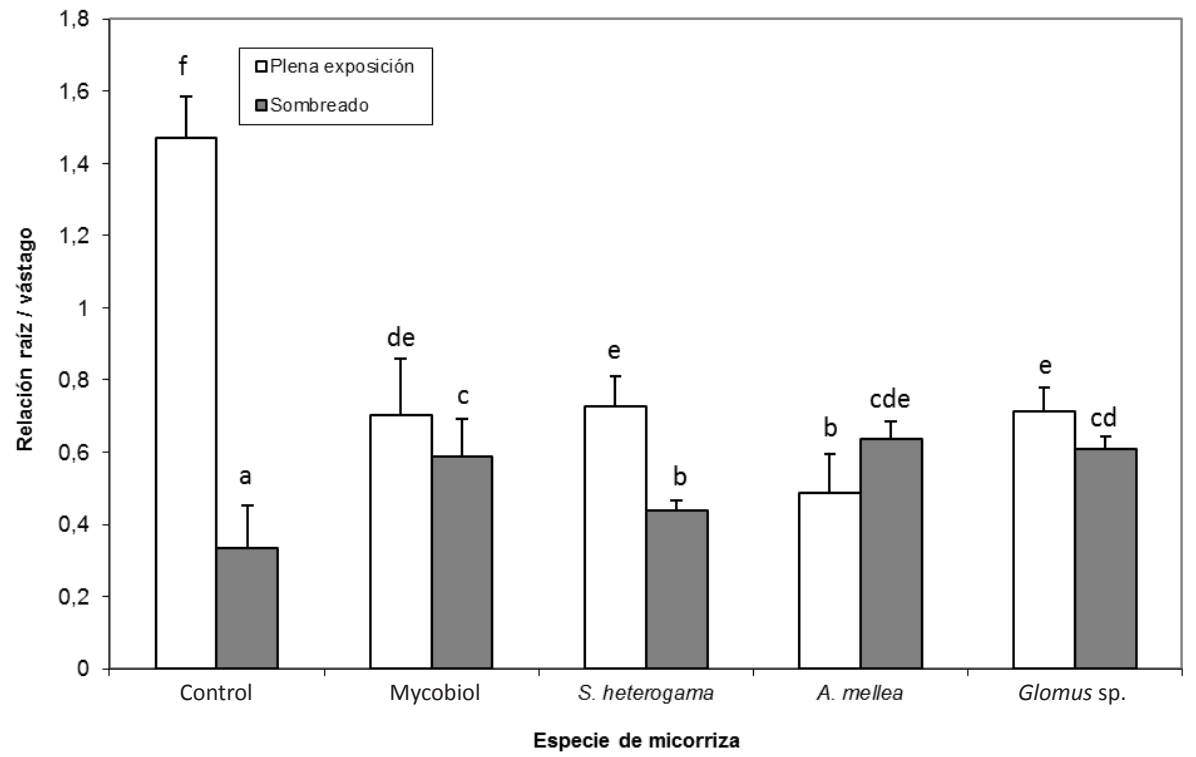

Figura 3. Relación Raíz/Vástago en plantas de lulo (Solanum quitoense Lam.), inoculadas con diferentes especies de micorrizas y expuestas a dos niveles de iluminación. 
sauce en respuesta a condiciones controladas de restricción hídrica y lumínica en tres niveles: plena exposición, sombra moderada ( $24,9 \%$ de reducción de luz) y sombra severa $(90 \%$ de reducción de luz), al inicio del establecimiento. La TCR en altura y en diámetro mostró diferencias significativas a favor de los tratamientos de sombra moderada y plena exposición, con respecto a los tratamientos bajo sombra severa. El mantenimiento del crecimiento en altura bajo sombra moderada y el aumento del área foliar y del área foliar específica, en ambos niveles de sombra, indican que estos clones de sauce tienen capacidad de aclimatación frente a la baja disponibilidad lumínica (Lambers et al. 1998), independientemente del nivel de disponibilidad hídrica que recibieron (Guarnaschelli et al. 2006); sin embargo, es evidente que para las plantas de lulo, esta capacidad de aclimatación es moderada y, en condiciones de restricción severa de luz, los parámetros de crecimiento se ven afectados.

La tasa de crecimiento absoluto (TCA), se redujo con la sombra, en $37,5 \%$ y, a su vez, las micorrizas también afectaron esta variable, con un incremento de $188,8 \%$, en relación con las plantas control, con el uso de S. heterogama, 155,5\% con Glomus sp., 133,3\% con Mycobiol y, por último, 116,6\% con A. mellea (Figura 4). Bajo las condiciones propias del
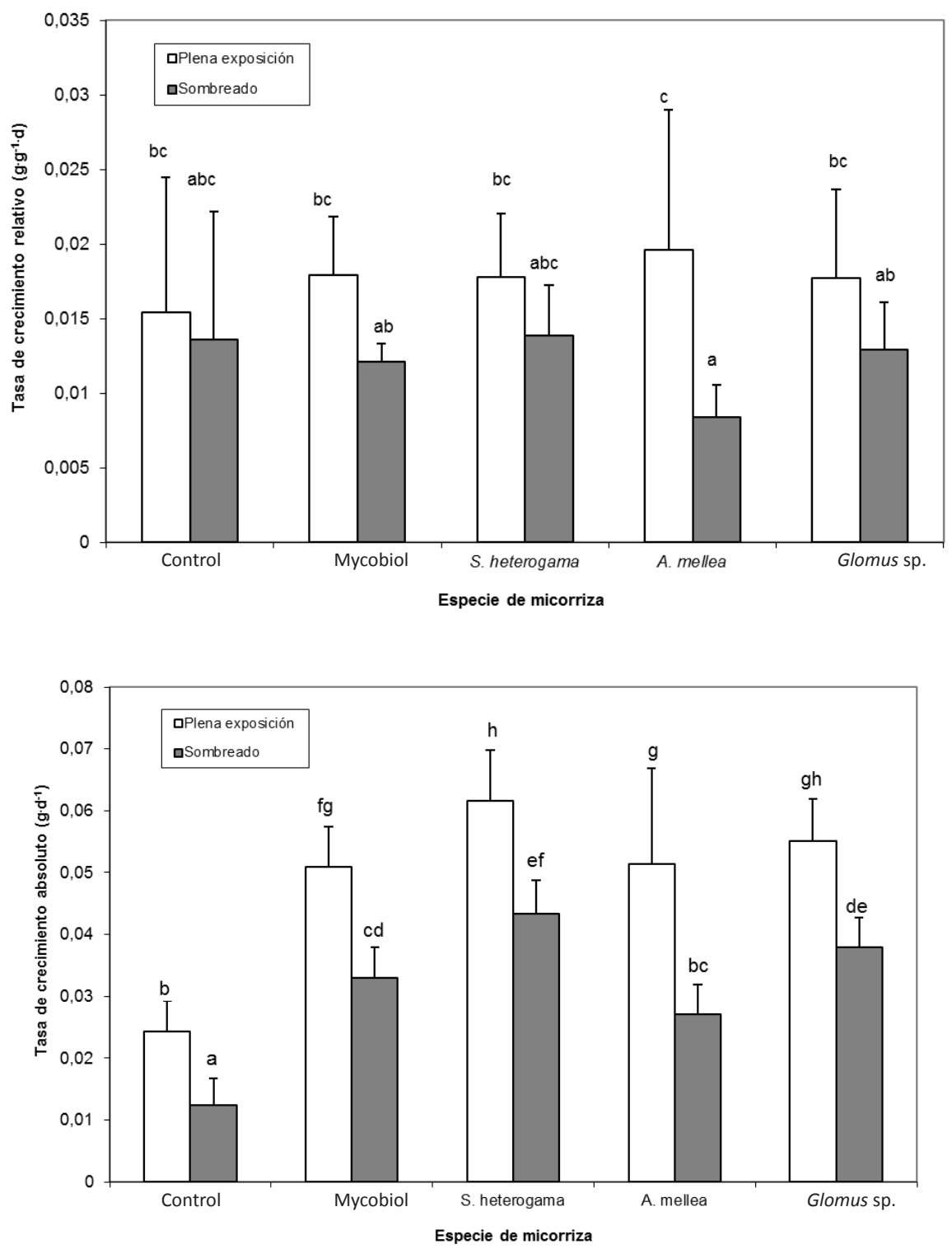

Figura 4. Tasa de crecimiento absoluto y relativo en plantas de lulo (Solanum quitoense Lam.), inoculadas con diferentes especies de micorrizas y expuestas a dos niveles de iluminación. 
presente ensayo, las plantas crecieron en contenedores individuales, sin experimentar la interferencia o competencia con otros individuos, lo cual, facilitó la expresión de los tratamientos a evaluar. Esta abstracción facilitó la expresión del crecimiento de las plantas expuestas a los diferentes tratamientos y es así, como en todos los casos, las plantas tratadas con micorrizas evidenciaron tasas de crecimiento absoluto mayores que los testigos, independientemente de la exposición a la luz. Moreira-Souza \& Cardoso (2002) realizaron trabajos en diferentes bosques de Brasil, con el objetivo de identificar la diversidad de los hongos micorrizógenos en dichos bosques y hallaron una mayor tasa de crecimiento inicial en los distintos árboles nativos con presencia de micorrizas arbusculares en la raíz, con lo cual, afirmaron que el éxito de los programas de reforestación, en gran medida, puede depender de la colonización micorrízica de la raíz de las plántulas, lo que incrementa su competitividad, debido al aumento en la tasa de crecimiento inicial. Esto hace suponer que en condiciones de sotobosque las micorrizas puedan fortalecer la competitividad de las plantas de lulo, como consecuencia de un incremento en la intensidad de su crecimiento.

En lo que respecta a la tasa de asimilación neta (TAN), se redujo en un $27,5 \%$, con la sombra; sin embargo, con respecto a las plantas control, las micorrizas indujeron un incremento en el valor de esta variable, en términos de $400 \%$ con S. heterogama, 328,5\% con Glomus sp., 285,7\% con A. mellea y $271,4 \%$ con Mycobiol (Figura 5). Esta alta tasa fotosintética, se puede justificar con base en elevadas concentraciones de fósforo foliar y en modificaciones en la hidratación de las hojas, los cuales, son producto del flujo de nutrimentos por parte del hongo hacia la planta, flujo que es compensado por la movilización del carbono en sentido basípeto. Sin lugar a dudas, la eficiencia fotosintética de las plantas micorrizadas, concuerda con la superioridad de las mismas sobre las plantas testigo en la totalidad de las variables evaluadas, mostrando un buen equilibrio de acuerdo con lo descrito por Jakobsen (1995), quien señala que el crecimiento de las plantas micorrizadas va a depender del balance entre el aporte de nutrientes, por parte del hongo y el aporte de carbono, por parte de la planta.

No se encontró diferencia significativa de los factores evaluados ni de su interacción sobre el número de esporas por gramo de suelo seco. Estos resultados concuerdan con otras investigaciones realizadas en frutales andinos, como mora y uchuva (Roveda et al. 2009), debido a que el incremento en las poblaciones de esporas en el suelo esta determinada por los ciclos de esporulación del micelio extraradical de las

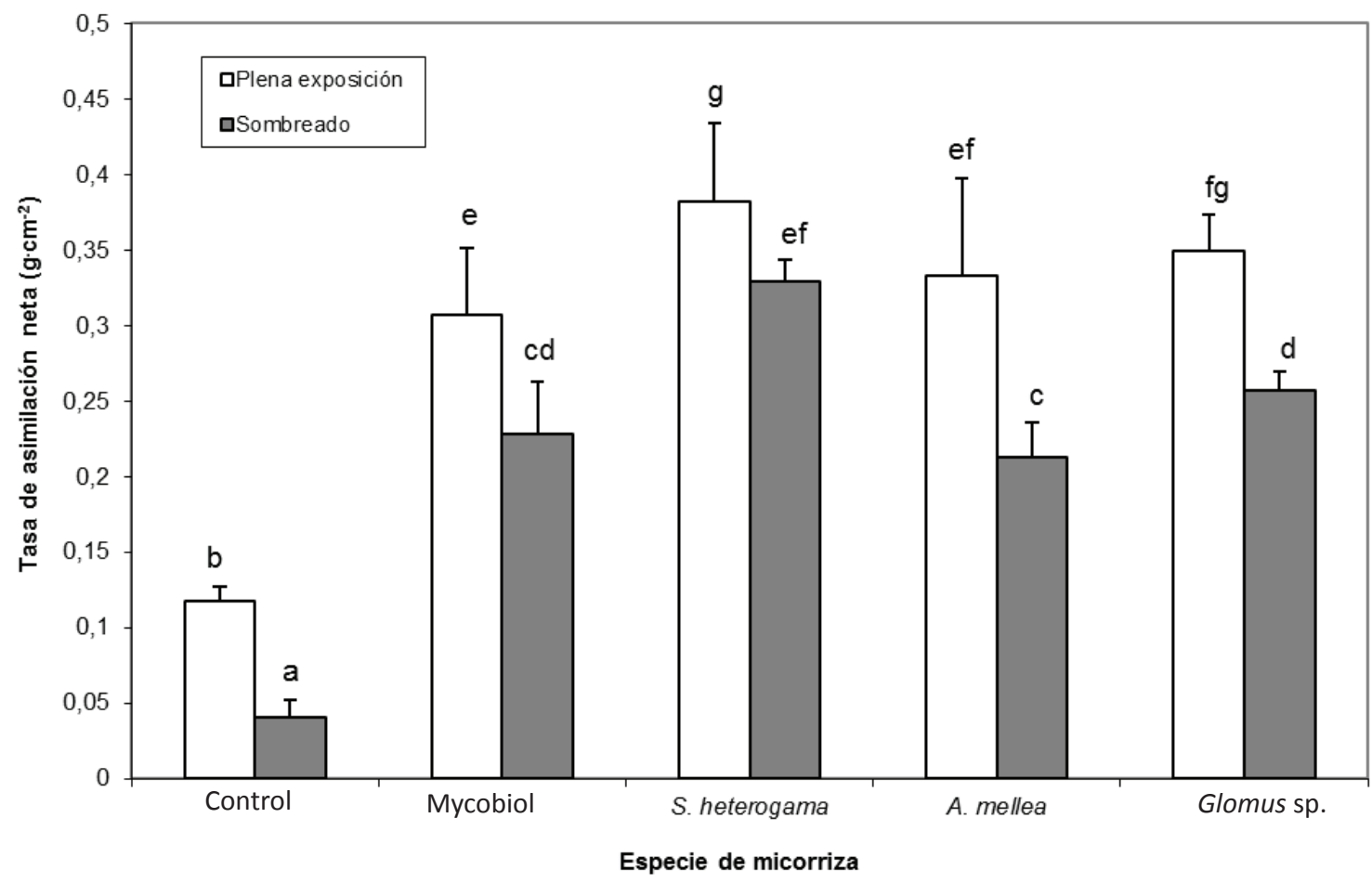

Figura 5. Tasa de asimilación neta en plantas de lulo (Solanum quitoense Lam.), inoculadas con diferentes especies de micorrizas y expuestas a dos niveles de iluminación. 
micorrizas arbusculares, que puede ser inducida en condiciones de estrés hídrico. De igual forma, no existe una relación entre las poblaciones de micorrizas en el suelo y los beneficios que genera en la planta.

De acuerdo con los resultados, en la totalidad de los parámetros evaluados, la sombra ejerció una influencia negativa sobre el crecimiento y el desarrollo de las plantas de lulo y debido a que esta planta predomina en el sotobosque es aconsejable la inoculación con hongos formadores de micorrizas, para corregir tal efecto. Todos los HFMA mostraron beneficios en la planta, expresados en las variables evaluadas y dentro de ellos, se destacaron las inoculaciones con $S$. heterogama.

Agradecimientos: Este estudio se desarrolló con el apoyo de la Corporación Colombiana de Investigación Agropecuaria (Corpoica), C.I. Tibaitatá y de la Dirección de Investigaciones (DIN) de la Universidad Pedagógica y Tecnológica de Colombia (UPTC), en el marco del plan de trabajo del grupo de investigación Ecofisiología Vegetal, del programa de Ingeniería Agronómica de la Facultad de Ciencias Agropecuarias. Conflictos de intereses: El manuscrito fue preparado y revisado con la participación de todos los autores, quienes declaramos que no existe ningún conflicto de intereses que ponga en riesgo la validez de los resultados presentados.

\section{BIBLIOGRAFÍA}

1. ALARCÓN, A.; FERRERA-CERRATO, R. 1999. Manejo de la micorriza arbuscular en sistemas de propagación de plantas frutícolas. Terra 17(3):179-191.

2. BAYLIS, G.T.S. 1967. Experiments on the ecological significance of Phycomycetous mycorrhizas. New Phytol. 66:231-243.

3. BOULLARD, B. 1960. La lumiere et les mycorrhizes. Ann. Biofog. 36:231-48.

4. CARDONA, D.; SADEGHIAN, S. 2005. Beneficios del sombrío de guamo en suelos cafeteros. CENICAFÉ, Av. Técn. Cenicafé. 335:1-5.

5. GERDEMANN, J.W.; NICOLSON, T.H. 1963. Spores of mycorhizal Endogone species extracted from soil by wet sieving and decanting. Trans. Brit. Mycol. Soc. 46:235-244.

6. GUARNASCHELLI, A.B.; GARAU, A.M.; CACCIA, F. 2006. Respuestas al déficit hídrico y lumínico durante el establecimiento de Salix matsudana x Salix alba 'A 13/44'. En: Actas Jornadas de Salicáceas. Buenos Aires. p.274-281.
7. GIANINAZZI-PEARSON, V.; AZCÓN-AGUILAR, C. 1991. Fisiología de las micorrizas vesiculo-arbusculares. En: Olivares, J.; Barea J.M. (eds): Fijacion y movilizacion biologica de nutrientes. Vol. II. (Fijacion de N y micorrizas). Consejo Superior de Invest. Cient. Madrid. p.175-202.

8. HARRIS, D.; PACOVSKY, R.S.; PAUL, E.A. 1985. Carbon economy of soybean-Rhizobium-Glomus associations. New Phytol. 73:71-80.

9. HEPPER, C. 1977. A colorimetric method for estimating vesicular-arbuscular mycorrhizal infection in roots. J. Soil Biol. Biochem. 9(1):15-18.

10. HUNT, R. 1990. Basic growth analysis. Plant growth analysis for beginners. Unwin Hyman. Boston. 110p.

11. JAKOBSEN, I. 1995. Transport of phosphorus and carbon in VA mycorrhizas. En: Varma, A.; Hock, B. (eds). Mycorrhiza: structure, function, molecular biology and biotechnology. Springer-Verlag, Berlin. p.297-324.

12. JARAMILLO, R.A.; GÓMEZ, G.L. 1989. Microclima en cafetales a libre exposición solar y bajo sombrío. Rev. CENICAFÉ. 40(3):65-79.

13. JOHNSON, P.N. 1976. Effects of soil phosphate level and shade on plant growth and mycorrhizas. N. Z. J. Bot. 14:333-40.

14. LAMBERS, H.; CHAPIN, III F.S.; PONS, T.J. 1998. Plant Physiological Ecology. Springer-Verlag, New York. 540p.

15. LOBO, M. 1991. Perspectivas de siembra del lulo o naranjilla (Solanum quitoense Lam). Universidad Nacional de Colombia. Facultad de Ciencias Agropecuarias, Palmira. Boletín Técnico. 2(2):125-130.

16. LOBO, M. 2000. Papel de la variabilidad genética en el desarrollo de los frutales andinos como alternativa productiva. Memorias 3er Seminario de Frutales de Clima Frío Moderado. Centro de Desarrollo Tecnológico de Frutales, Manizales. p.27-36.

17. MARSCHNER, H. 1991. Mechanisms of adaptation of mycorrhizal (Glomus fasiculatum) influence on plants to acid soils. Dev. Plant Soil Sci. 45:683-702.

18. MCGEE, P.A.; FURBY, J.H. 1992. Formation and structure of mycorrhizas of seedlings of coachwood (Ceratopetalum apetalum). Austral. J. Bot. 40:291-304. 
19. MEDINA, C.I.; SÁNCHEZ, D.; CAMAYO, G.; LOBO, M.; MARTÍNEZ, E. 2008. Anatomía foliar comparativa de materiales de lulo (Solanum quitoense Lam.) con y sin espinas. Rev. Corpoica. 9(1):5-13.

20. MENGE, J.A. 1983. Utilization of vesicular-arbuscular extension of the phosphorus depletion zone in mycorrhizal fungi in agriculture. Can. J. Bot. 61:10151024.

21. MOREIRA-SOUZA, M.; CARDOSO, E.J.B.N. 2002. Dependencia micorrízica de Araucaria angustifolia (Bert.) O. Ktze. sob doses de fósforo. Rev. Bras. Ciência do Solo. 26:905-912.

22. NEHLS, U.; HAMPP, R. 2000. Carbon allocation in ectomycorrhizas. Physiol. Mol. Plant Pathol. 57:95-100.

23. NIEMI, K.; JULKUNEN-TIITTO, R.; TEGELBERG, R.; HÄGGMAN, H. 2005. Light sources with different spectra affect root and mycorrhiza formation in scots pine in vitro. Tree Physiol. 25:123-128.

24. PAZ, I.E.; SÁNCHEZ DE PRAGER, M. 2007. Relación entre la longitud de micelio externo de hongos micorrízicos y algunas propiedades del suelo bajo dos sistemas de sombrío en café, meseta de Popayán, Colombia. Rev. Biotecn. Sector Agrop. Agroind. 5(1):64-69.

25. PENG, S.; EISSENSTAT, D.M.; GRAHAM, J.H.; WILLIAMS, K.; HODGE, N.C. 1993. Growth depression in mycorrhizal citrus at high-phosphorus supply. Plant Physiol. 101:1063-1071.

26. PEREIRA, G.; SÁNCHEZ, M.; RÍOS, D.; HERRERA, M.A. 2001. Micorrizas vesículo arbusculares y su incidencia en el crecimiento de plántulas de Eucalyptus camaldulensis Den. Bosque. 22(2):39-44.

27. ROSA, T.M.D.L.; APHALO, P.J.; LEHTO, T. 1998. Effects of far-red light on the growth, mycorrhizas and mineral nutrition of scots pine seedlings. Plant Soil. 201:17-25.

28. ROSA, T.M.D.L.; LEHTO, T.; APHALO, P.J. 1999. Does far-red light affect growth and mycorrhizae of Scots pine seedlings growing in forest soil? Plant Soil. 211:259-268.
29. ROVEDA, G.; CABRA, L.; RAMÍREZ, M.M.; PEÑARANDA, A. 2007. Efecto de las micorrizas arbusculares sobre la aclimatación y endurecimiento de microplántulas de mora (Rubus glaucus). Rev. Corpoica. 8(1):28-36.

30. ROVEDA, G.; RAMÍREZ, M.M.; CABRA, L.; PEÑARANDA, A. 2009. Biofertilización en el cultivo de la mora. En: Barrero, L. (ed) Caracterización, evaluación y producción de material limpio de mora con alto valor agregado. Corpoica. Produmedios. p.43-56.

31. SCHULZE, E.D.; BECK, E.; MÜLLER-HOHENSTEIN, K. 2002. Plant Ecology. Springer Verlag. Heidelberg. p.23-44.

32. SÁNCHEZ DE PRAGER, M. 1999. Endomicorrizas en agroecosistemas colombianos. Universidad Nacional, Palmira. 226p.

33. SIQUEIRA, J.O.; SAGGIN-JÚNIOR, O.J.; FLORESAYLAS, W.W.; GUIMARÃES, P.T.G. 1998. Arbuscular mycorrhizal inoculation and superphosphate application influence plant development and yield of coffee in Brazil. Mycorrhiza. 7(6):293-300.

34. SMITH, S.; READ, D. 1997. Mycorrhizal symbiosis. $2^{\text {nd }}$ ed. Academic Press, San Diego, 605p.

35. SPRUGEL, D.G.; BROOKS, J.R.; HINCKLEY, T.M. 1996. Effects of light on shoot geometry and needle morphology in Abies amabilis. Tree Physiol. 16:91-98.

36. SWISHER, M. 1999. Manual para los estudios de campo, Módulo 1 La Ecología de la Parcela. Universidad de la Florida. 84p.

37 TURK, M.A.; ASSAF, T.A.; HAMEED, K.M.; AL-TAWAHA, A.M. 2006. Significance of mycorrhizae. World J. Agr. Sci. 2(1):16-20.

38. WRIGHT, D.P.; SCHOLES, J.D.; READ, D.J.; ROLFE, S.A. 2000. Changes in carbon allocation and expression of carbon transporter genes in Betula pendula Roth. colonized by an ectomycorrhizal fungus Paxillus involutus (Batsch). Fr. Plant Cell Environ. 23:39-49.

Recibido: Febrero 19 de 2011

Aceptado: Noviembre 16 de 2012 